\title{
PREVENTING RAT-TAILED MAGGOT INCURSION INTO DAIRY SHEDS
}

\author{
D.J. WILSON ${ }^{1}$, P.J. GERARD ${ }^{1}$ and J.E. DE VILLIERS ${ }^{2}$ \\ ${ }^{1}$ AgResearch, Private Bag 3123, Hamilton, New Zealand \\ ${ }^{2}$ University of Waikato, Hamilton, Private Bag 3150, New Zealand \\ Corresponding author: derrick.wilsonl@agresearch.co.nz.
}

\begin{abstract}
Rat-tailed maggots are the larvae of hover flies (Diptera: Syrphidae), of which there are about 40 species in New Zealand, many of them native. The adults are important pollinators and the larvae live in water and mud, feeding on rotting organic matter. High concentrations occur in dairy effluent bunkers and become health and sanitary issues when prepupal larvae seeking suitable pupation sites exit bunkers and invade nearby dairy sheds. Replicated small scale model bunkers were set up to test barrier methods that could prevent maggots entering sensitive areas and divert them to suitable pupation sites. Known numbers of migratory prepupal larvae were placed in the model bunkers and their movements recorded over a timed period. Both round piping and angular strips proved effective barriers, while soil, sand and sawdust were all equally attractive as a pupation medium. Keywords: rat-tailed maggot, Eristalis tenax, herd home, barrier, substrate.
\end{abstract}

\section{INTRODUCTION}

Hover flies (Diptera: Syrphidae) are important pollinators and can often be seen hovering around flowers in urban and rural areas. There are about 40 species in New Zealand, many of them native. The larvae live in stagnant water, ditches and effluent ponds, feeding on microbes and decaying organic material. The larvae breathe through a long narrow tube attached to their rear, earning their common name of "rat-tailed maggots'. When fully mature, the prepupal larvae exit the liquid material to pupate in surrounding soil and vegetation.

Hover fly larvae are currently causing problems in herd homes, a recent innovation in the New Zealand dairy industry. Herd homes are open-walled animal shelters used to protect animals from excess heat or cold, and to hold and feed herds when pastures are at risk of pugging. Effluent is collected beneath the grated floor and later spread on pastures as fertiliser when there is no danger of nutrient leaching. A new configuration currently being tested is linking a shelter to the dairy shed yarding. However, incursions of the migrating prepupal larvae into sensitive dairy shed areas have put any further development of this configuration on hold. Hover fly larvae can spread diseases to animals and people, including pathogenic mycobacteria that cause paratuberculosis (Fischer et al. 2005).

Initial observations by the authors indicated hover fly larvae were excellent climbers, could move rapidly (>1 m/min), and could climb $4 \mathrm{~cm}$ vertical bands of Fluon ${ }^{\circledR}$, a fluocarbon-based polymer coating that prevents most insects from crossing. A lowmaintenance, pesticide-free solution was sought that would be compatible with the shelter operations. This paper reports on investigations into the species present in the shelter bunkers, barrier methods to direct hover fly larvae away from dairy sheds and substrates that may encourage cessation of movement and initiation of pupation. 


\section{Species assessment}

\section{MATERIALS AND METHODS}

Samples of larvae were collected from a shelter bunker at Parakao, Northland, in mid December 2008, and from Ruakaka, Northland, in January 2009. Pupae were collected from four further Northland sites during February 2009. The initial pupae were held in individual vials at $20^{\circ} \mathrm{C}$. However, survival was poor and subsequent pupae were reared to adult stage by placing them in shallow soil on a tray inside a plastic bag at ambient temperatures in the shade. Adults were identified by comparing morphological characters with pinned Syrphidae in the Ruakura collection.

\section{Barrier assessment}

In a small scale test, two barriers were selected for testing with the dual aims of encouraging migrating larvae to move sideways along the barriers and if a larva did attempt to cross them, it was likely to fall back into the bunker. Barrier A was a $4.5 \mathrm{~cm}$ plastic pipe cut lengthwise to form a $\mathrm{C}$ shape in cross section. Barrier B was a flatbottomed V-shaped plastic electrical conduit that was $1.5 \times 2.0 \times 1.5 \mathrm{~cm}$ in cross-section. The test arenas (Fig. 1) were two transparent plastic cubes $35 \times 32 \times 27 \mathrm{~cm} \mathrm{high,} \mathrm{each}$ fitted with one of the two barriers. The barriers were firmly attached to all four internal sides, joined neatly at the corners $18 \mathrm{~cm}$ above the base, with the open side facing the centre of the arena. At both ends of one barrier in each cube, there was an exit hole in the adjoining wall, $1.5 \mathrm{~cm}$ in diameter, to which $120 \mathrm{ml}$ specimen vials containing soil were connected to capture any exiting larvae. Effluent from a shelter bunker was placed at the base of each arena to a depth of $1 \mathrm{~cm}$ to provide a medium for the test larvae.

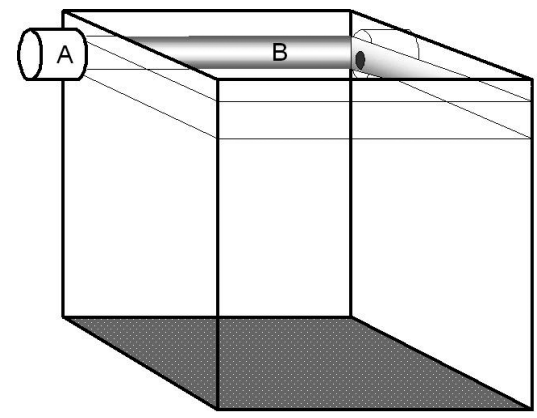

\section{FIGURE 1: Diagram of test arena showing position of collection vials (A)} and barriers (B).

Effluent containing hover fly larvae was collected from a Waikato herd home bunker and kept in vented plastic containers at ambient temperature until required. On each experimental run, 10 active prepupal larvae were placed carefully in the base effluent of each arena. Larvae activity was recorded every $5 \mathrm{~min}$ for the first hour, and at 3 and $24 \mathrm{~h}$ with the number of hits (larvae touching barrier), larvae under the barrier, on the walls and larvae exited to the soil in vials noted. This was replicated eleven times.

A larger-scale barrier experiment was established using a disused goat milking parlour. A tray $60 \times 90 \times 5 \mathrm{~cm}$ high was placed on the ground at the junction of two concrete walls. Uncut plastic pipe, $6.5 \mathrm{~cm}$ in diameter, was mounted firmly to both walls $90 \mathrm{~cm}$ above the tray. On the two remaining sides, walls were constructed of solid waterproof hardboard securely affixed to the tray and concrete walls at the edges. To these walls, strips of the previously-used flat-bottomed V-shaped conduit were mounted, hollow side out, at the same height as the round pipe barrier. Plastic bottles containing soil were attached at the end walls of the pipe barriers to contain exiting larvae. Thirty prepupal 
larvae were released into the effluent tray and escape attempts (effluent trails) recorded over a $24 \mathrm{~h}$ period. This was repeated three times.

\section{Pupation substrate preference}

Cubes as used above were divided into quarters with hardboard partitions $6 \mathrm{~cm}$ high. Each quarter was filled with either one of four substrates: moist sand; moist field soil; moist coarse sawdust; or a single layer of moist sacking on a hard corflute surface fixed level to the top of the partition. There were 4 replicates placed in a Latin square design to ensure no substrate occupied the same location in any one cube. A $50 \mathrm{ml}$ plastic specimen vial was positioned on the centre partition meeting point of each cube and 5 active prepupal larvae added. The cubes were held in shade at ambient temperatures and covered with gauze to keep out birds. After $72 \mathrm{~h}$, the cubes were assessed for pupa location and depth located in substrate. All pupae were removed and retained to adult emergence.

\section{Statistical analyses}

Barrier experimental data were analysed using ANOVA (GenStat 10.2). Substrate experimental data were analysed using Fisher's exact $2 \times 2$ test of numbers versus equal choice.

\section{Species assessment}

\section{RESULTS}

Over 150 hover fly pupa were collected in Northland and sent to the laboratory. While many pupae failed to develop into adult flies, of those that successfully emerged, all were Eristalis tenax (Linnaeus). Likewise, all adults emerging from locally-sourced populations used in the barrier and substrate trials were E. tenax.

\section{Barrier assessment}

Over the course of the small scale cube barrier experiment, no prepupal hover fly larvae crossed either of the barrier types even though barrier hits averaged around 30 for both in the first hour (Table 1). Larvae successfully exited into collection vials and there was no significant difference between barrier types in this regard.

The mean number of barrier hits in the large scale trial was less than 25 in the $24 \mathrm{~h}$ observation period. Again no larvae escaped from the test arena.

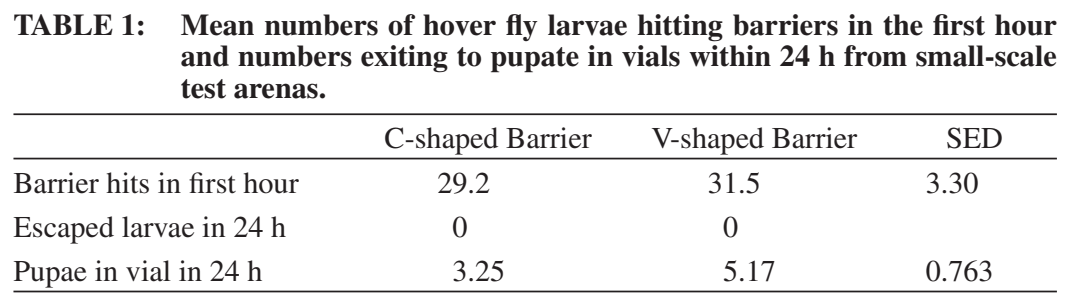

\section{Pupation substrate preference}

In the substrate preference trials (Table 2), the moist sack was chosen significantly less often as a suitable pupation medium over the other three substrates. It was observed by the authors that the larvae buried themselves rapidly in the sawdust.

TABLE 2: Percentage of hover fly pupae found after $72 \mathrm{~h}$ when given choice of four pupation substrates.

\begin{tabular}{lcccc}
\hline & \multicolumn{4}{c}{ Substrate } \\
\cline { 2 - 5 } & Sack & Sand & Soil & Sawdust \\
\hline Percent chosen & 2.7 & 34.3 & 28.8 & 34.3 \\
Fisher's exact $2 \times 2$ test & $\mathrm{P}<0.001$ & $\mathrm{P}=0.124$ & $\mathrm{P}=0.598$ & $\mathrm{P}=0.124$ \\
\hline
\end{tabular}




\section{DISCUSSION}

The only hover fly species collected, E. tenax, is cosmopolitan in distribution. The females must feed on pollen to complete reproduction and both sexes feed on nectar (Gilbert 1986). The adults are attracted to yellow flowers, leading to their importance in the pollination of yellow-flowered crops (Ilse 1949). The migrating larvae can cause problems, such as contamination of livestock feed, short circuits from accumulations in electrical boxes and congregations in unwanted places (Pfiester \& Kaufman 2009).

The results from these barrier studies demonstrate that even a reasonably small barrier can prevent larval movement from a containment area and divert larvae to a collection point. The flat-bottomed V-shaped barrier was easier to join at corners than the pipe barriers, but did not provide as good a channel for larvae to crawl along. In a working bunker, the barriers would need to be larger than those tested to ensure any larvae pupating under them didn't form a blockage or bridge that could allow larvae to cross the barrier. Also, the C-shaped pipe may provide better protection from manure accumulation than the more open V-shape. Further work would need to be done with design engineers to incorporate a within-bunker barrier that is robust enough to withstand normal farm operations. The ability to divert and collect pupating larvae raises the possibility of herd homes shelters providing a potential source of large numbers of E. tenax pupae for crop pollination.

An alternative solution to the problem of hover fly larvae migrating to dairy sheds is to ensure liquid effluent does not accumulate in bunkers close by (Fonterra regulations stipulate effluent ponds must be at least $45 \mathrm{~m}$ away). Therefore, if a shelter is to be built within this limit, draining away slurry and/or using mulches, to ensure the formation of compost rather than slurry, could be incorporated in the bunkers. This in turn may eliminate the hoverfly larval problem.

Other methods used overseas to prevent prepupal movement include using pesticides and dry earth barriers (Townsend 2005). However, using insecticides poses the risk of residues in milk. In contrast, dry earth or substrate barriers are "environmentally friendly", and are simple and inexpensive systems away from wash-down areas. A single layer of moist sack on a hard surface proved unattractive as a pupation site, while moist coarse sawdust, sand and soil were equally attractive.

In conclusion, problems with hover fly larvae can be prevented by using barriers to block entry into and direct them away from sensitive areas. Additionally, herd homes shelter effluent bunkers could prove excellent sources of pollinators in rural communities.

\section{ACKNOWLEDGEMENTS}

We thank Catherine Cameron (AgResearch) for statistical analyses, J W Pouls from Ohaupo for local supply of rat-tailed maggots, and Jenny Dymock and Tom Pow for Northland samples. This study was funded by Herd Homes® Systems Limited.

\section{REFERENCES}

Fischer OA, Matlova L, Dvorska L, Svastova P, Bartos M, Weston RT, Kopecna M, Trcka I, Pavlik I 2005. Potential risk of Mycobacterium avium subspecies paratuberculosis spread by syrphid flies in infected cattle farms. Medical and Veterinary Entomology 19: 360-366.

Gilbert FS 1986. Hoverflies, Naturalists' Handbooks 5. Cambridge University Press, Cambridge. $67 \mathrm{p}$.

Ilse D 1949. Colour discrimination in the drone fly Eristalis tenax. Nature 163: 255-256.

Pfiester M, Kaufman PE.2009. Drone fly, rat-tailed maggot Eristalis tenax (Linnaeus) (Insecta: Diptera: Syrphidae). University of Florida IFAS Extension EENY-445 http://edis.ifas.ufl.edu/IN809 (retrieved 29 March 2009).

Townsend L 2005. Manure flies: Rat-tailed maggots and moth flies. ENTFACT 500, University of Kentucky, USA. http://www.ca.uky.edu/entomology/entfacts/ef500.asp (retrieved 8 April 2009). 